\title{
Decreased expression of CD63 tetraspanin protein predicts elevated malignant potential in human esophageal cancer
}

\author{
XIAOJING LAI $^{1 *}$, QING GU$^{1 *}$, XIA ZHOU $^{1}$, WEI FENG $^{1}$, XIAO LIN $^{1}$, YAN HE $^{2}$, \\ JINMING CAO ${ }^{2}$, PENGFEI LIU ${ }^{3}$, HUOJUN ZHANG $^{4}$ and XIAO ZHENG ${ }^{1}$
}

\begin{abstract}
${ }^{1}$ Department of Radiation Oncology, Zhejiang Key Laboratory of Diagnosis and Treatment Technology on Thoracic Oncology (Lung and Esophagus), Zhejiang Cancer Hospital (Zhejiang Cancer Research Institute), Hangzhou, Zhejiang 310022;

${ }^{2}$ School of Radiation Medicine and Protection and Jiangsu Provincial Key Laboratory of Radiation Medicine and Protection, Medical College of Soochow University, Soochow, Suzhou 215123; ${ }^{3}$ Department of Gastroenterology, The Affiliated Jiangyin Hospital of Southeast University, Jiangyin, Jiangsu 214400; ${ }^{4}$ Department of Radiation Oncology, Shanghai Changhai Hospital, The Second Military Medical University, Shanghai 200433, P.R. China
\end{abstract}

Received August 19, 2015; Accepted February 13, 2017

DOI: $10.3892 / 01.2017 .6023$

\begin{abstract}
The tetraspanin CD63 has been described to have critical roles in multiple biological processes, including tumorigenesis and metastasis in several types of cancer. However, its role in esophageal carcinoma (EC) has not been reported. In the current study, immunohistochemistry was used to investigate CD63 expression in 106 esophageal cancer samples, 49 adjacent esophagus tissues and 17 normal esophagus mucosa tissues. The results revealed that the overexpression of CD63 was observed in esophageal cancer samples and negatively correlated with tumor stage and lymph node metastasis. To further evaluate the role of CD63 in esophageal carcinoma, the invasiveness of EC cells was analyzed using matrigel invasion assays and wound healing assays in vitro. Furthermore, it was found that CD63 knockdown increased the invasiveness of TE- 1 cells through the upregulation of matrix metalloproteinase (MMP) expression via promoting epithelial-mesenchymal transition. The current data therefore
\end{abstract}

Correspondence to: Dr Huojun Zhang, Department of Radiation Oncology, Shanghai Changhai Hospital, The Second Military Medical University, 168 Changhai Road, Shanghai 200433, P.R. China

E-mail: chyyzhj@163.com

Dr Xiao Zheng, Department of Radiation Oncology, Zhejiang Key Laboratory of Diagnosis and Treatment Technology on Thoracic Oncology (Lung and Esophagus), Zhejiang Cancer Hospital (Zhejiang Cancer Research Institute), 1 East Banshan Road, Gongshu, Hangzhou, Zhejiang 310022, P.R. China

E-mail: zhengxiao@zjcc.org.cn

*Contributed equally

Key words: esophageal carcinoma, tetraspanin CD63, tumor stage, lymph nodes metastasis, invasiveness, epithelial-mesenchymal transition suggested that low levels of CD63 expression may be involved in the tumor progression of esophageal carcinoma.

\section{Introduction}

Esophageal cancer is the eighth most common malignant cancer in incidence and the sixth in cancer mortality worldwide (1). In China, esophageal cancer is the fourth most common cause of cancer-associated mortalities, and the majority of cases are squamous cell carcinoma (SCC) (2). Generally, patients with esophageal cancer are diagnosed at a relatively late stage and have a very poor prognosis. The 5 -year survival rate of esophageal cancer is $<10 \%(3,4)$. The most important prognostic factors in esophageal carcinoma include the extent of the primary tumor and the metastasis of lymph nodes (5). An increasing number of metastatic lymph nodes are associated with a progressively poorer prognosis (6). The identification of more efficient biomarkers associated with lymph node metastasis is important for predicting the clinical outcomes in esophageal cancer.

CD63, also known as lysosome-associated membrane glycoprotein 3, melanoma-associated antigen ME491 or melanoma-associated antigen MLA1, is a member of the transmembrane-4 superfamily (TM4SF; tetraspan proteins), and is a cell surface glycoprotein (7-9). TM4SF molecules mediate signal transduction events that have a role in the regulation of cellular processes, including growth, adhesion motility and differentiation, and has been associated with tumor progression in human non small cell lung cancer, prostate cancer, breast cancer, astrocytomas, pancreatic cancer and melanoma (10-13). The tetraspanin CD63 has been implicated in metastatic signaling pathways in several cancer types. Kondoh et al (14) suggested that CD63 may act as an anti-metastatic gene in human malignant melanoma. The precancerous lesions of melanoma exhibit intense CD63 expression, whereas the tissues from the melanomas with the dermis invasion or with distant metastasis are weakly stained (14). Radford et al (15) confirmed the suppressive role of CD63 in 
melanoma invasion and progression, due to its association with $\beta 1$ integrins and their interaction with specific extracellular matrix (ECM) substrates (fibronectin, laminin, and collagen). Sordat et al (16) has reported that substrate-immobilized anti-CD63 antibodies are able to enhance colon carcinoma cell migration and invasion. Seubert et al (17) revealed that CD63 overexpression enhanced the tumor cell-intrinsic metastatic potential by initiating $\beta$-catenin-dependent epithelial-mesenchymal transition (EMT), which contrasted the results of other studies. Kwon et al (18) suggested that CD63 negativity is able to predict poor prognosis for early-stage lung adenocarcinoma (15). Jang et al (19) reported that reduced CD63 expression contributes to the invasive and metastatic ability of human melanoma cells due to the increased cell motility, matrix proteolytic capability and ability to detach from the surrounding matrix. The results reported by Toricelli et al (20) revealed that a supramolecular complex containing CD63, tissue inhibitor of metalloproteinase-1 (TIMP-1) and $\beta 1$-integrins increased anoikis resistance in melanoma cells by activating Phosphatidylinositol-4,5-bisphosphate 3-kinase signaling pathway, independently of protein kinase B phosphorylation.

Previous studies have suggested that the tetraspanin family may be involved in the metastasis of esophageal cancers. For example, TM4SF3, a member of the tetraspanin family, has been reported to be a pro-metastatic factor in esophageal carcinoma via upregulating ADAM12 m expression (21). Ectopic expression of the tetraspanin cell surface receptor uroplakin $1 \mathrm{~A}$ inhibited cell proliferation, clonogenicity, cell motility and tumor formation by inhibiting nuclear translocation of $\beta$-catenin in esophageal carcinoma cells (21). Inactivation of its downstream targets, including Cyclin-D1, c-Jun, c-Myc, and matrix metalloproteinase 7 (MMP-7) (22). However, the role of the TM4SF member CD63 in esophageal cancer remains unclear.

The current study examined the expression of CD63 in primary esophageal cancer to examine its association with tumor stage and lymph node metastasis. The invasiveness of esophageal cancer cell lines following downregulation of CD63 expression was also investigated. In addition, the underlying mechanisms by which CD63 affects the invasiveness of esophageal carcinoma cells were examined.

\section{Materials and methods}

Tissue samples. A total of 106 esophageal carcinoma (EC) samples and 49 matched adjacent esophagus tissues were collected by endoscopic biopsies or surgical resection from 106 patients (Table I), and 17 normal esophagus mucosa tissues were obtained from surgical resections of trauma patients. The age of the EC patients ranged between 32 and 78, and the mean age was 59.8. The age of the trauma patients ranged from 32 to 72 , and the mean age was 57.2. No patient had received any other treatment prior to surgery. These tissues were obtained from the Gastrointestinal Center at the Jiangyin People's Hospital, Medical School of University of Southeast of China (Jiangyin, China) from February 2010 to December 2012. This study was performed under the approval the Ethics Committees of Jiangyin People's Hospital and the patients gave written informed consent to participate.
Table I. Clinicopathological data from 106 patients with EC.

Clinicopathological factor

No. of patients $(\%)$

Sex

Male

$62(58.48)$

Female

$44(41.52)$

Histologic type

$\mathrm{SqC}$

58 (54.72)

$\mathrm{AdC}$

Stage

Early (I/II)

$68(64.15)$

Advanced (III/IV)

$38(35.85)$

Tumor status ${ }^{\mathrm{a}}$

$\mathrm{T} 1$

$18(16.98)$

$\mathrm{T} 2$

$38(35.85)$

T3

$22(20.75)$

$\mathrm{T} 4$

$28(26.42)$

Lymph node metastasis ${ }^{\mathrm{b}}$

N0

$64(60.38)$

N1

27 (25.47)

7 (6.60)

$\mathrm{N} 2$

$8(7.55)$

Distant metastasis

Negative

$104(98.1)$

Positive

2 (1.9)

${ }^{\text {aT }}$ Tumor $(\mathrm{T})$ categories according to the Union for International Cancer Control (UICC) Tumor Node Metastasis (TNM) classification of

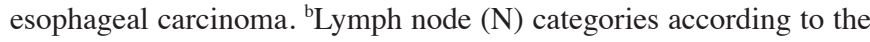
UICC TNM classification of esophageal carcinoma. sqC, squamous cell carcinoma; AdC, adenocarcinoma.

All diagnoses were based on pathological and/or cytological evidence. According to the classification criteria from the World Health Organization (23), the histological features of the specimens were evaluated by two senior pathologists. For immunohistochemistry, tissues were frozen in liquid nitrogen and maintained at $-80^{\circ} \mathrm{C}$ immediately following endoscopic biopsies or surgical resection.

Immunohistochemistry. The expression patterns of CD63 in human esophageal cancer samples, the adjacent normal esophagus samples and the normal esophagus mucosa tissues were analyzed using immunohistochemistry. Sections of formalin-fixed and paraffin-embedded tissue with a thickness of $6 \mu \mathrm{m}$ were deparaffinized and heated in citrate buffer (pH 6.0; Sigma-Aldrich; Merck KGaA, Darmstadt, Germany) at $95^{\circ} \mathrm{C}$ for $30 \mathrm{~min}$ as an antigen retrieval protocol. Endogenous peroxidase activity was blocked with $0.3 \%$ hydrogen peroxide, and non-specific-binding sites were reduced by incubating the sections at room temperature with $4 \%$ skim milk powder for $30 \mathrm{~min}$. The sections were then incubated overnight at $4^{\circ} \mathrm{C}$ with a rabbit monoclonal antibody to CD63 protein (cat. no. ab134045; dilution, 1:1,000; Abcam, Cambridge, MA USA). Biotinylated secondary antibody mouse anti-rabbit 
immunoglobulin G-B (cat. no., sc-2491; dilution, 1:1,000, Santa Cruz Biotechnology, Santa Cruz, CA, USA) was then added to the sections followed by incubation for $30 \mathrm{~min}$ at room temperature. An avidin-biotin-peroxidase complex (Beyotime Institute of Biotechnology, Nantong, China) was added for an additional $30 \mathrm{~min}$. Following treatment with a 3,3'-diaminobenzidine substrate-chromogen system (Dako; Agilent Technologies Inc., Santa Clara, CA, USA) for $8 \mathrm{~min}$, counterstaining was performed with hematoxylin. All histological assessments were performed by the same pathologist. For statistical analysis, the sections with $<10 \%$ of the whole tissue mass stained were classified as negative; the sections with $10-25 \%$ of the whole tissue mass stained were classified as weakly positive $(+1)$; the sections with $25-75 \%$ of the whole tissue mass stained were defined as moderately positive (+2); the sections with $>75 \%$ of the tissue stained positive were regarded as strongly positive $(+3)$.

Cell culture and transfection. The human esophageal cancer cell line TE-1 was cultured in Dulbecco's modified Eagle's medium (Thermo Fisher Scientific Inc., Waltham, MA, USA) supplemented with $10 \%$ fetal bovine serum (FBS; Gibco; Thermo Fisher Scientific, Inc.) and antibiotics (100 U/ml penicillin and $100 \mu \mathrm{g} /$ streptomycin, Sigma-Aldrich; Merck $\mathrm{KGaA}$ ). Cells were grown at $37^{\circ} \mathrm{C}$ in a $5 \% \mathrm{CO}_{2}$ atmosphere and were transfected according to the manufacturer's protocol using Lipofectamine 2000 (Invitrogen; Thermo Fisher Scientific, Inc.) with CD63 siRNA (Santa Cruz Biotechnology Inc., Dallas, TX, USA).

Western blot analysis. Esophageal carcinoma TE-1 cells were divided into three groups: Control (with no transfection protocol), NC (cells transfected with empty plasmid), and siCD63 (cells transfected with small interfering RNA targeting CD63). Following treatment, cells were lysed with radioimmunoprecipitation assay lysis buffer $(50 \mathrm{mM}$ Tris- $\mathrm{HCl}$, pH 7.4; 150 mM NaCl; $1 \%$ Triton X-100; $1 \%$ sodium deoxycholate; 0.1\% SDS) and protease inhibitor cocktail (P8340; Sigma-Aldrich; Merck $\mathrm{KGaA}$ ) for $30 \mathrm{~min}$ at $4^{\circ} \mathrm{C}$. BCA protein assay (Bio-Rad Laboratories, Hercules, CA, USA) was used to determine the protein concentration. Equal quantities of protein lysates (40 $\mu \mathrm{g}$ total protein) were electrophoretically separated by $10 \%$ SDS-PAGE and transferred to polyvinylidene difluoride membranes (EMD Millipore, Billerica, MA, USA). Following blocking with $5 \%$ nonfat milk in TBST (20 mM Tris, pH 7.5; $500 \mathrm{mM} \mathrm{NaCl} ; 0.05 \%$ Tween-20) for $1 \mathrm{~h}$ at room temperature, the membranes were then incubated overnight at $4^{\circ} \mathrm{C}$ with the primary antibody to CD63 (cat. no., ab134045; dilution, 1:1,000, Abcam), E-cadherin (cat. no., sc-59778; dilution, 1:200, Santa Cruz Biotechnology), vimentin (cat. no. sc-6260; dilution, 1:200, Santa Cruz Biotechnology), MMP-2 (cat. no. sc-13594; dilution, 1:200, Santa Cruz Biotechnology) and MMP-9 (cat. no. sc-21733; dilution, 1:200, Santa Cruz Biotechnology). $\beta$-Actin (cat. no. sc-8432; dilution, 1:200, Santa Cruz Biotechnology) was used as a loading control. Following washing four times with TBST, the membranes were incubated at room temperature with a horseradish peroxidase-conjugated secondary antibody (cat. nos. sc-2005, sc-2357; dilution, 1:1,000, Santa Cruz Biotechnology) for $2 \mathrm{~h}$, and visualized by chemiluminescence using an enhanced chemiluminescence system (GE Healthcare Life Sciences, Chalfont, UK).

In vitro migration assay. Esophageal carcinoma TE-1 cells were seeded in 6-well plates and allowed to reach a confluent state, then were divided into three groups: Control (with no transfection protocol), NC (cells transfected with empty plasmid), and siCD63 (cells transfected with small interfering RNA targeting CD63). Following treatment, the monolayer was scratched with the tip of a $200 \mu l$ pipette. The floating and detached cells were removed by washing twice with PBS. Subsequently, fresh serum-free medium was added. At 0 and $24 \mathrm{~h}$, images were captured to assess the extent of cell migration using an Olympus BX 40 Light Microscope (Olympus Corporation, Tokyo, Japan).

In vitro invasion assay. The invasive potential of TE-1 cells was assessed using 24-well Matrigel invasion chambers (pore size $8 \mu \mathrm{m}$, Corning Costar Corporation, Corning, NY, USA). Inserts were pre-coated with $40 \mu \mathrm{l}$ Matrigel (1:4 dilution; BD Biosciences, San Jose, CA, USA). Then, $5 \times 10^{4}$ cells/ ml pre-transfected with CD63 siRNA in serum-free medium were added to the upper chambers. The lower chambers contained $500 \mu \mathrm{l}$ medium supplemented 10\% FBS. Following incubation for $24 \mathrm{~h}$ at $37^{\circ} \mathrm{C}$, the cells remaining in the upper chambers were removed with PBS-moistened cotton swabs, and the invading cells on the underside of the insert filter were fixed with $3.7 \%$ paraformaldehyde for $30 \mathrm{~min}$ at room temperature and stained with Giemsa for $1 \mathrm{~h}$ at room temperature. Images of 5 random microscopic fields gathered by an Olympus BX 40 Light Microscope (Olympus Corporation) were captured to calculate the average number of invaded cells.

Statistical analysis. Mean \pm standard error of the mean of several independent experiments were calculated. One-way analysis of variance was applied to analyze the data, and then the significance of differences among different groups was evaluated by Student-Newman-Keuls post hoc test. Statistical tests were performed using Graph-Pad PRISM version 6.0 (Graph-Pad Software, San Diego, CA). P<0.05 was considered to indicate a statistically significant difference.

\section{Results}

Expression of CD63 is upregulated in esophageal cancer tissues. To investigate the variation in expression levels between the normal and cancerous esophageal tissues, CD63 protein expression levels were evaluated by immunohistochemistry staining in 106 paraffin-embedded human esophageal cancer tissues, 49 adjacent esophagus tissues and the 17 normal esophagus mucosa tissues. Overall positive staining for CD63 was frequently observed in the cytoplasm of EC tissues, whereas no staining of CD63 was observed in the adjacent esophagus tissues and the normal esophagus mucosa tissues (Fig. 1A). The sections were subsequently scored by a pathologist; there were significant differences in CD63 expression between esophageal cancer tissues, adjacent esophagus tissues and the 17 normal esophagus mucosa tissues (Fig. 1B, $\mathrm{P}<0.01$ ). This demonstrated that CD63 expression 

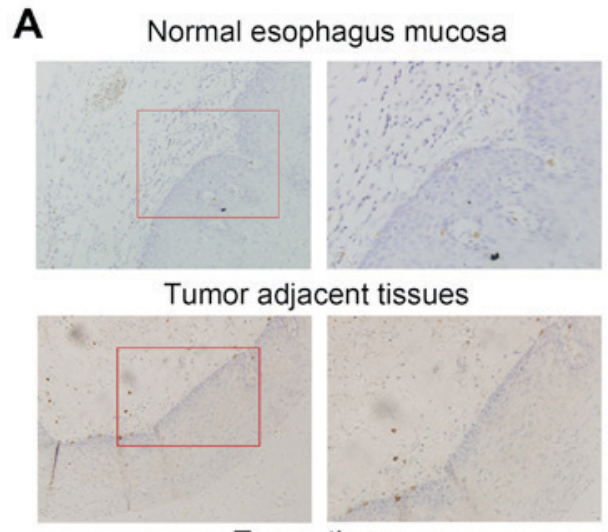

Tumor tissues

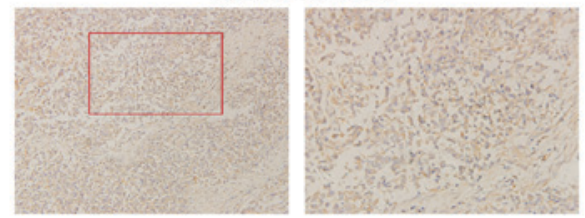

B

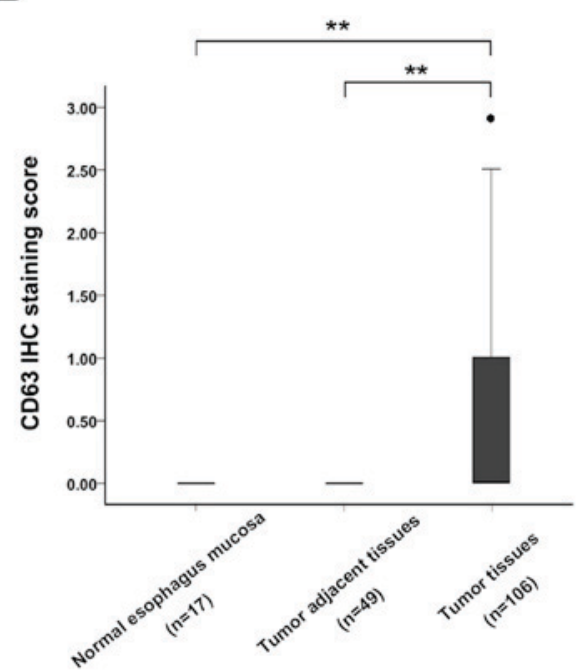

Figure 1. (A) Representative IHC analysis of CD63 expression in human esophageal cancer tissues, adjacent esophagus tissues and 17 normal esophagus mucosa tissues. (B) Bar graph representing the range of CD63 IHC staining score in esophageal cancer tissues ( $\mathrm{n}=106)$, matched adjacent esophagus tissues $(\mathrm{n}=49)$ and normal esophagus mucosa tissues $(\mathrm{n}=17),{ }^{* *} \mathrm{P}<0.01$. IHC, immunohistochemistry.

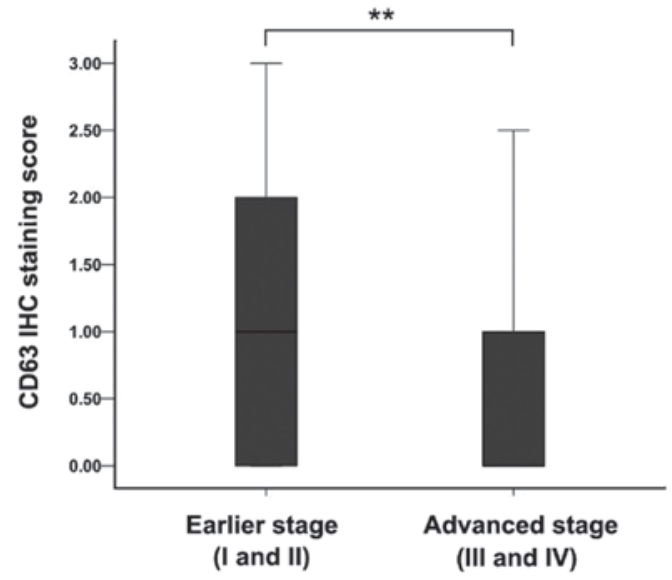

Figure 2. Bar graph representing the range of CD63 IHC staining scores in earlier stage (I and II) and advanced stage (III and IV) esophageal carcinoma tissues. ${ }^{* *} \mathrm{P}<0.01$. IHC, immunohistochemistry.

was increased in esophageal carcinoma and may be a regulator in the development of EC.

Association between CD63 expression and clinicopathological factors. The CD63 expression differences in the EC tissues in different clinical stages were subsequently analyzed. The average CD63 expression level in earlier stage (I and II) esophageal carcinoma was higher than the level in advanced stage (III and IV) (Fig. 2; P<0.01). CD63 expression levels were also found to be associated with lymph node metastasis in EC cases. Weak positive staining of EC tissues was detected in patients with lymph node metastasis. However, in the patients without metastatic lymph nodes, the CD63 staining was strongly positive (Fig. 3 ). This suggests that CD63 may have an important role in the lymph node metastasis of EC.

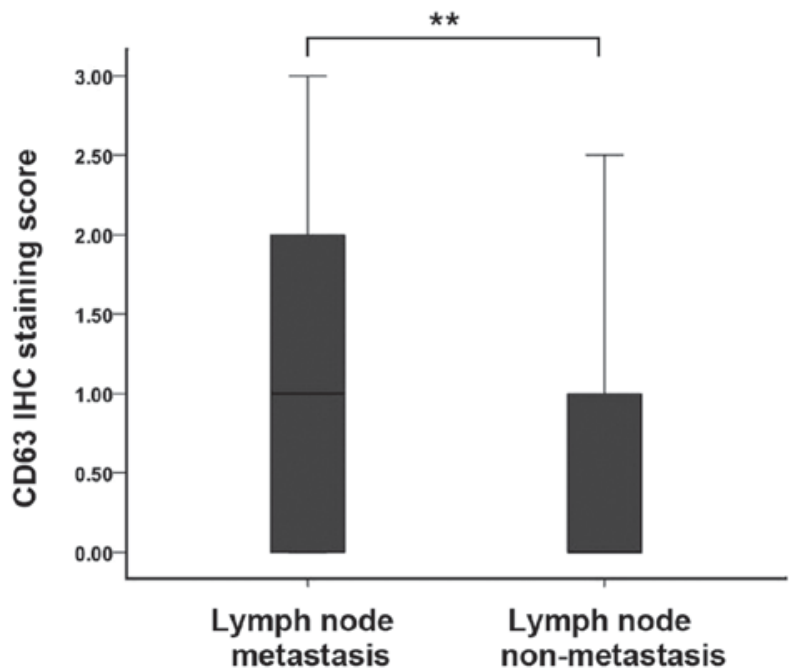

Figure 3. Bar graph representing the range of CD63 IHC staining score in EC tissues with or without metastatic lymph nodes. ${ }^{* *} \mathrm{P}<0.01$. IHC, immunohistochemistry.

Downregulation of CD63 promotes migration of EC cells. To investigate the role of reduced CD63 expression in the migration of EC, the esophageal cancer cells TE-1 were transfected with CD63-silencing RNA. Western blot analysis revealed that $\mathrm{CD} 63$ protein expression was markedly reduced by the siRNA transfection (Fig. 4A). Matrigel invasion assays were performed to explore the effect of CD63 on the invasiveness of TE-1 cells. As presented in Fig. 4B and C, the ability of TE-1 cells to invade through the Matrigel and membrane was increased by CD63 knockdown when compared with the control cells or the empty plasmid transfected cells. The CD63 siRNA transfected TE-1 cells exhibited a 2-fold higher invasiveness than the control cells. In vitro wound healing assays were performed to detect the motility of TE-1 cells, 
A

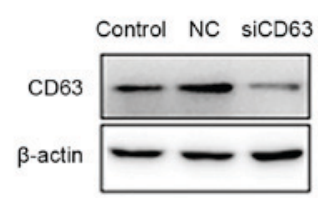

B

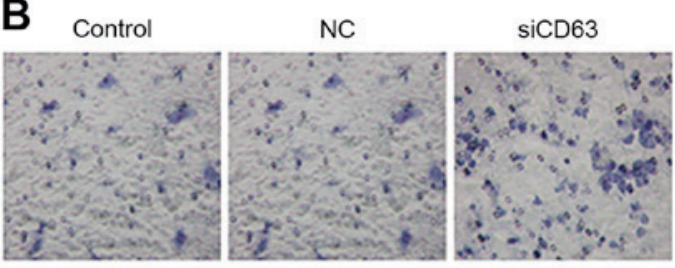

C

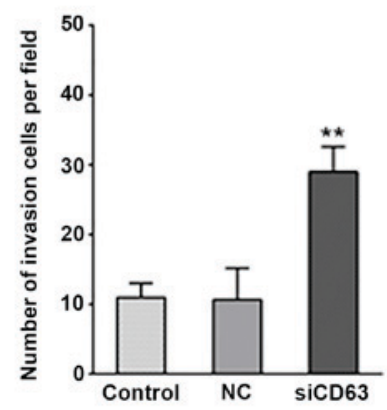

D

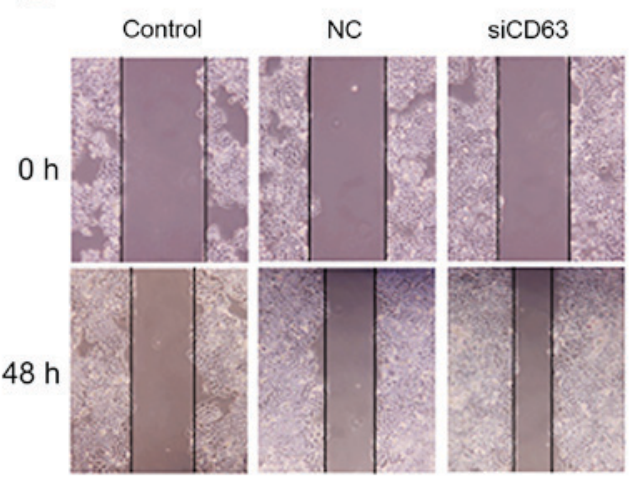

E

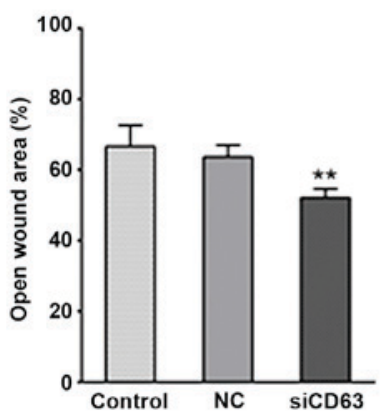

Figure 4. Effect of CD63 on esophageal cancer cell invasiveness. (A) Western blot analysis confirmed a marked reduction of CD63 protein following CD63 small interfering RNA transfection in esophageal cancer cells. (B and C) In the Matrigel invasion assay, following treatment for $48 \mathrm{~h}$, the number of CD63 knockdown TE-1 cells that migrated to the bottom surface of the membrane was greater compared with TE-1 cells transfected with empty plasmid and the control TE-1 cells. (D and E) Wound healing was observed $48 \mathrm{~h}$ after treatment, and the open wound area of CD63 knockdown TE-1 cells was larger than the TE-1 cells transfected with empty plasmid and the control TE-1 cells. ${ }^{* *} \mathrm{P}<0.01$. NC, negative control; siCD63, small interfering RNA targeting CD63.

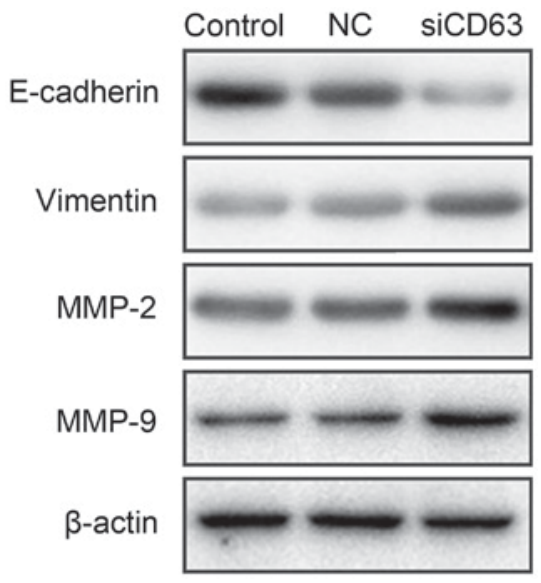

Figure 5. Western blot analysis of the expression of E-cadherin, vimentin, MMP-2 and MMP-9 following treatment with small interfering RNA targeting CD63. The epithelial marker E-Cadherin was downregulated and the mesenchymal maker vimentin was upregulated by CD63 knockdown. MMP-2 and MMP-9 expression levels were increased by CD63 knockdown. MMP, matrix metalloproteinase; NC, negative control; siCD63, small interfering RNA targeting CD63.

which is another important characteristic of metastatic cells. As presented in Fig. 4D and E, CD63 knockdown noticeably reduced the wound area, suggesting that CD63 knockdown enhanced the migration capability of esophageal cancer.

CD63 knockdown enhances invasiveness of EC cells via promoting EMT. To explore the mechanisms underlying the effect of CD63 on the invasiveness of EC, TE-1 cells were transfected with CD63 siRNA or empty plasmid. After $48 \mathrm{~h}$ of incubation, western blotting assays were used to detect the expression of EMT-associated proteins and MMPs. E-cadherin was established as the epithelial maker, and vimentin as the mesenchymal maker. As presented in Fig. 5, TE-1 cells transfected with CD63 siRNA exhibited downregulation of E-cadherin expression and upregulation of vimentin when compared with the control cells or the empty plasmid transfected cells. The matrix metalloproteinase MMP-2 and MMP-9 expression was increased by CD63 knockdown. These results suggest that CD63 knockdown regulates the invasiveness of esophageal cancer cells via promoting EMT.

\section{Discussion}

The prognosis of esophageal carcinoma remains poor despite advances in aggressive treatment for EC $(3,4)$, due to late diagnosis and the rapid metastasis of cancer cells $(24,25)$. The present study investigated CD63 as a potential marker for the migration capability of esophageal carcinoma.

The TM4SF is a group of cell surface proteins with four transmembrane domains. CD63 was the first tetraspanin to be characterized and it was found to be located on human chromosome 12q13 (26). Various studies have demonstrated that CD63 regulates intracellular transport and localization by interacting with a number of proteins $(27,28)$. CD63 is a member of the tetraspanin proteins, and is a cell surface glycoprotein. CD63 has been reported to be upregulated in 
breast cancer, astrocytoma and melanoma, and is involved in various biological processes $(18,26,29)$. In the present study, the protein levels of CD63 in EC tissues were revealed to be significantly increased compared with adjacent tumor tissues or the normal esophagus mucosa tissues. Furthermore, the tetraspanin CD63 has been suggested to be associated with the biological behavior of solid tumors, and has particularly been implicated in metastatic signaling pathways (30). The hematogenous metastasis of CD63-negative human melanoma cells in nude mice may be reduced by CD63 transfection (15). Kwon et al (18) suggested that CD63 negativity has a potential as a biomarker for predicting poor prognosis in earlier stage lung adenocarcinoma. Jung et al (31) identified CD63 as a cell surface-binding partner for TIMP-1, regulating the polarization and survival of breast epithelial cells. However, the expression pattern and clinicopathological roles of CD63 have not been investigated in EC in previous studies. In the current study, CD63 negativity was significantly associated with advanced tumor stage and a greater number of metastatic lymph nodes in EC. Intensely immunostained samples were identified in earlier stage (I and II) EC. In advanced stage (III and IV) EC, the samples were weakly immunostained. A previous study has demonstrated that CD63 expression is weaker as the stage advances in melanoma (14), consistent with the findings of the current study in esophageal cancer. Lymph node metastasis is one of the most important prognostic factors in EC, and the number of metastatic lymph nodes is consistently associated with the long-term outcomes of patients with EC (6). Regarding a potential correlation with lymph node metastasis, the current study revealed that CD63 protein expression was weaker as the number of metastatic lymph nodes increased. To further elucidate the role of CD63 in EC progression, wound healing migration and matrigel invasion assays were performed to detect the invasiveness of the TE-1 esophageal cancer cell line. Following siRNA-induced CD63 knockdown, the invasiveness of TE-1 cells was enhanced. In the current study, CD63 suppressed the metastasis of EC cells. In a previous study, CD63 was originally described as being involved in cancer metastasis (30). Tominaga et al (32) suggested that RPN2-mediated CD63 glycosylation regulates breast cancer cell malignancy, including drug resistance and invasion. Jang et al (19) reported that reduced CD63 expression contributes to the invasive and metastatic ability of human melanoma cells, due to increased cell motility, matrix proteolytic capability and ability to detach from the surrounding matrix. CD63 has also been implicated in regulating the functions of the tumor development-associated protein, membrane-associated type-1 MMP in ECM turnover, thereby increasing cell invasiveness and metastasis (33). The association between CD63 and integrins, including $\alpha 4 \beta 1$, $\alpha 3 \beta 1, \alpha 6 \beta 1$, LFA- 1 and $\beta 2$, was reported to be involved in increased tumor cell motility and metastasis, which mediates binding to the ECM (34-37). CD63 potentially interacts with numerous other proteins such as CD9, CD81 and $\beta 1$ integrins, contributing to the downstream cell signaling pathway (38). The association between CD63 and $\beta 1$ integrins has been observed in human melanoma cells, and integrins were described as having major roles in the invasive capacity acquisition of cancer cells (30). TIMP-1 was identified to regulate the integrin signaling complex via its interaction with CD63 on the cell surface (31). CD63 associated with CD9 has also been revealed to suppress the motility and metastasis of mouse melanoma cells by downregulation of CD9 expression (39). It has been reported that a complex in the surface membrane of melanoma cells formed by CD63, CD9 and CD81 was involved in signal transduction (38). Seubert et al (17) reported that CD63 increased the tumor cell intrinsic metastatic potential by initiating $\beta$-catenin-dependent EMT and then affecting cell plasticity in human ovarian carcinoma, human gastric carcinoma and mouse melanoma cells. The current study identified the pro-metastatic role of downregulated CD63 expression in esophageal cancer, and examined the underlying mechanism. In addition, the epithelial marker E-cadherin was downregulated and the mesenchymal maker vimentin was upregulated by CD63 knockdown, suggesting that CD63 may function as a potent inhibitor of metastasis in EC via regulating the EMT. MMP family proteins are involved in the breakdown of ECM and participate in the process of cancer metastasis (40). A number of studies have reported that EMT-inducing transcription factors are directly or indirectly involved in cancer cell metastasis through various signaling cascades, with the ultimate consequence of the upregulation of metastatic proteins, including MMPs (41). The present study identified that MMP-2 and MMP-9 expression levels were increased by CD63 knockdown, suggesting that CD63 knockdown-induced EC invasion may be due to MMP-2 and MMP-9 overexpression through the activation of EMT signal pathway. However, the underlying mechanism by which CD63 activates EMT in EC remains to be investigated.

In conclusion, to the best of our knowledge the present study revealed for the first time that CD63 expression was upregulated in EC tissues, and was negatively correlated with tumor stage and lymph node metastasis. CD63 knockdown enhanced EC cell invasion, and was mediated by MMP-2 and MMP-9 overexpression through the activation of EMT signaling pathway. This indicates that CD63 may be a promising biomarker to predict the risk of EC progression.

\section{Acknowledgements}

The present study was supported by grants from the Zhejiang Medical Science and Technology Foundation (grant nos. 2014KYA007 and 2014KYA009).

\section{References}

1. Torre LA, Bray F, Siegel RL, Ferlay J, Lortet-Tieulent J and Jemal A: Global cancer statistics, 2012. CA Cancer J Clin 65: 87-108, 2015.

2. Pennathur A, Gibson MK, Jobe BA and Luketich JD: Oesophageal carcinoma. Lancet 381: 400-412, 2013.

3. Dipetrillo T, Suntharalingam M, Ng T, Fontaine J, Horiba N, Oldenburg N, Perez K, Birnbaum A, Battafarano R, Burrows W and Safran H: Neoadjuvant paclitaxel poliglumex, cisplatin, and radiation for esophageal cancer: A phase 2 trial. Am J Clin Oncol 35: 64-67, 2012.

4. van Hagen P, Hulshof MC, van Lanschot JJ, Steyerberg EW, van Berge Henegouwen MI, Wijnhoven BP, Richel DJ, Nieuwenhuijzen GA, Hospers GA, Bonenkamp JJ, et al: Preoperative chemoradiotherapy for esophageal or junctional cancer. N Engl J Med 366: 2074-2084, 2012.

5. Eloubeidi MA, Desmond R, Arguedas MR, Reed CE and Wilcox CM: Prognostic factors for the survival of patients with esophageal carcinoma in the U.S.: The importance of tumor length and lymph node status. Cancer 95: 1434-1443, 2002. 
6. Cho JW, Choi SC, Jang JY, Shin SK, Choi KD, Lee JH, Kim SG, Sung JK, Jeon SW, Choi IJ, et al: Lymph node metastases in esophageal carcinoma: An Endoscopist's view. Clin Endosc 47: $523-529,2014$

7. Maecker HT, Todd SC and Levy S: The tetraspanin superfamily: Molecular facilitators. FASEB J 11: 428-442, 1997.

8. Hunziker W and Geuze HJ: Intracellular trafficking of lysosomal membrane proteins. Bioessays 18: 379-389, 1996.

9. Atkinson B, Ernst CS, Ghrist BF, Herlyn M, Blaszczyk M, Ross AH, Herlyn D, Steplewski Z and Koprowski H: Identification of melanoma-associated antigens using fixed tissue screening of antibodies. Cancer Res 44: 2577-2581, 1984.

10. Hemler ME: Tetraspanin proteins mediate cellular penetration, invasion and fusion events and define a novel type of membrane microdomain. Annu Rev Cell Dev Biol 19: 397-422, 2003.

11. Hemler ME, Mannion BA and Berditchevski F: Association of TM4SF proteins with integrins: Relevance to cancer. Biochim Biophys Acta 1287: 67-71, 1996.

12. Hölters S, Anacker J, Jansen L, Beer-Grondke K, Dürst M and Rubio I: Tetraspanin 1 promotes invasiveness of cervical cancer cells. Int J Oncol 43: 503-512, 2013.

13. Kwon MS, Shin SH, Yim SH, Lee KY, Kang HM, Kim TM and Chung YJ: CD63 as a biomarker for predicting the clinical outcomes in adenocarcinoma of lung. Lung Cancer 57: 46-53, 2007.

14. Kondoh M, Ueda M, Ichihashi M and Mishima Y: Decreased expression of human melanoma-associated antigen ME491 along the progression of melanoma pre-canceroses to invasive and metastatic melanomas. Melanoma Res 3: 241-245, 1993.

15. Radford KJ, Mallesch J and Hersey P: Suppression of human melanoma cell growth and metastasis by the melanoma-associated antigen CD63 (ME491). Int J Cancer 62: 631-635, 1995.

16. Sordat I, Decraene C, Silvestre T, Petermann O, Auffray C, Piétu $\mathrm{G}$ and Sordat B: Complementary DNA arrays identify CD63 tetraspanin and alpha3 integrin chain as differentially expressed in low and high metastatic human colon carcinoma cells. Lab Invest 82: 1715-1724, 2002.

17. Seubert B, Cui H, Simonavicius N, Honert K, Schäfer S, Reuning U, Heikenwalder M, Mari B and Kruger A: Tetraspanin CD63 acts as a pro-metastatic factor via $\beta$-catenin stabilization. Int J Cancer 136: 2304-2315, 2015.

18. Kwon MS, Shin SH, Yim SH, Lee KY, Kang HM, Kim TM and Chung YJ: CD63 as a biomarker for predicting the clinical outcomes in adenocarcinoma of lung. Lung Cancer 57: 46-53, 2007.

19. Jang HI and Lee H: A decrease in the expression of CD63 tetraspanin protein elevates invasive potential of human melanoma cells. Exp Mol Med 35: 317-323, 2003.

20. Toricelli M, Melo FH, Peres GB, Silva DC and Jasiulionis MG: Timp1 interacts with beta-1 integrin and CD63 along melanoma genesis and confers anoikis resistance by activating PI3-K signaling pathway independently of Akt phosphorylation. Mol Cancer 12: 22, 2013.

21. Zhou Z, Ran YL, Hu H, Pan J, Li ZF, Chen LZ, Sun LC, Peng L, Zhao XL, Yu L, et al: TM4SF3 promotes esophageal carcinoma metastasis via upregulating ADAM12m expression. Clin Exp Metastasis 25: 537-548, 2008.

22. Kong KL, Kwong DL, Fu L, Chan TH, Chen L, Liu H, Li Y, Zhu YH, Bi J, Qin YR, et al: Characterization of a candidate tumor suppressor gene uroplakin 1A in esophageal squamous cell carcinoma. Cancer Res 70: 8832-8841, 2010.

23. Yamasaki M, Miyata H, Miyazaki Y, Takahashi T, Kurokawa Y, Nakajima K, Takiguchi S, Mori M and Doki Y: Evaluation of the nodal status in the 7th edition of the UICC-TNM classification for esophageal squamous cell carcinoma: Proposed modifications for improved survival stratification: Impact of lymph node metastases on overall survival after esophagectomy. Ann Surg Oncol 21: 2850-2856, 2014
24. D'Amico TA: Outcomes after surgery for esophageal cancer Gastrointest Cancer Res 1: 188-196, 2007.

25. Enzinger PC and Mayer RJ: Esophageal cancer. N Engl J Med 349: 2241-2252, 2003

26. Hotta $\mathrm{H}$, Ross AH, Huebner $\mathrm{K}$, Isobe $\mathrm{M}$, Wendeborn $\mathrm{S}$, Chao MV, Ricciardi RP, Tsujimoto Y, Croce CM and Koprowski H: Molecular cloning and characterization of an antigen associated with early stages of melanoma tumor progression. Cancer Res 48: 2955-2962, 1988

27. Lin D, Kamsteeg EJ, Zhang Y, Jin Y, Sterling H, Yue P, Roos M, Duffield A, Spencer J, Caplan M and Wang WH: Expression of tetraspan protein CD63 activates protein-tyrosine kinase (PTK) and enhances the PTK-induced inhibition of ROMK channels. J Biol Chem 283: 7674-7681, 2008.

28. Yoshida T, Ebina H and Koyanagi Y: N-linked glycan-dependent interaction of CD63 with CXCR4 at the Golgi apparatus induces downregulation of CXCR4. Microbiol Immunol 53: 629-635, 2009.

29. Sauer G, Kurzeder C, Grundmann R, Kreienberg R, Zeillinger R and Deissler $\mathrm{H}$ : Expression of tetraspanin adaptor proteins below defined threshold values is associated with in vitro invasiveness of mammary carcinoma cells. Oncol Rep 10: 405-410, 2003.

30. Radford KJ, Thorne RF and Hersey P: Regulation of tumor cell motility and migration by CD63 in a human melanoma cell line. J Immunol 158: 3353-3358, 1997.

31. Jung KK, Liu XW, Chirco R, Fridman R and Kim HR: Identification of CD63 as a tissue inhibitor of metalloproteinase-1 interacting cell surface protein. EMBO J 25: 3934-3942, 2006.

32. Tominaga N, Hagiwara K, Kosaka N, Honma K, Nakagama H and Ochiya T: RPN2-mediated glycosylation of tetraspanin CD63 regulates breast cancer cell malignancy. Mol Cancer 13: 134, 2014

33. Takino T, Miyamori H, Kawaguchi N, Uekita T, Seiki M and Sato H: Tetraspanin CD63 promotes targeting and lysosomal proteolysis of membrane-type 1 matrix metalloproteinase. Biochem Biophys Res Commun 304: 160-166, 2003.

34. Rubinstein E, Le Naour F, Lagaudrière-Gesbert C, Billard M, Conjeaud $\mathrm{H}$ and Boucheix C: CD9, CD63, CD81, and CD82 are components of a surface tetraspan network connected to HLA-DR and VLA integrins. Eur J Immunol 26: 2657-2665, 1996.

35. Berditchevski F, Zutter MM and Hemler ME: Characterization of novel complexes on the cell surface between integrins and proteins with 4 transmembrane domains (TM4 proteins). Mol Biol Cell 7: 193-207, 1996.

36. Berditchevski F, Bazzoni G and Hemler ME: Specific association of CD63 with the VLA-3 and VLA-6 integrins. J Biol Chem 270: 17784-17790, 1995.

37. Skubitz KM, Campbell KD, Iida J and Skubitz AP: CD63 associates with tyrosine kinase activity and CD11/CD18 and transmits an activation signal in neutrophils. J Immunol 157: 3617-3626, 1996.

38. Radford KJ, Thorne RF and Hersey P: CD63 associates with transmembrane 4 superfamily members, CD9 and CD81, and with beta 1 integrins in human melanoma. Biochem Biophys Res Commun 222: 13-18, 1996.

39. Si Z and Hersey P: Expression of the neuroglandular antigen and analogues in melanoma. CD9 expression appears inversely related to metastatic potential of melanoma. Int J Cancer 54 : 37-43, 1993.

40. Zucker S and Vacirca J: Role of matrix metalloproteinases (MMPs) in colorectal cancer. Cancer Metastasis Rev 23: 101-117, 2004.

41. Tania M, Khan MA and Fu J: Epithelial to mesenchymal transition inducing transcription factors and metastatic cancer. Tumour Biol 35: 7335-7342, 2014 